\title{
The EEE experiment: cosmic rays, multigap resistive plate chambers and high school students
}

\author{
M. Abbrescia*1 , S. Aiola ${ }^{2}$, R. Antolini ${ }^{3}$, C. Avanzini ${ }^{4}$, R. Baldini Ferroli ${ }^{5,6}$, \\ G. Bencivenni ${ }^{6}$, E. Bossini ${ }^{4}$, E. Bressan ${ }^{7}$, A. Chiavassa ${ }^{8}$, C. Cicalo ${ }^{9}$, L. Cifarelli ${ }^{7}$, \\ F. Coccetti ${ }^{3,5}$, E. Coccia ${ }^{3}$, D. De Gruttula ${ }^{10}$, S. De Pasquale ${ }^{10}$, A. Di Giovanni $^{3}$, \\ M. D'Incecco ${ }^{3}$, K. Doroud ${ }^{11}$, M. Dreucci ${ }^{6}$, F. L. Fabbri' ${ }^{6}$, V. Frolov ${ }^{8}$, M. Garbini ${ }^{7}$, \\ G. Gemme ${ }^{12}$, I. Gnesi ${ }^{8}$, C. Gustavino ${ }^{3}$, D. Hatzifotiadu' ${ }^{7,13}$, P. La Rocca ${ }^{2,5}$, S. Li ${ }^{11}$, \\ F. Librizzi ${ }^{2}$, A. Maggiora ${ }^{8}$, M. Massai ${ }^{4}$, H. Menghetti ${ }^{7}$, S. Miozzi ${ }^{6}$, R. Moro ${ }^{3}$, \\ M. Panareo ${ }^{14}$, R. Paoletti ${ }^{4}$, L. Perasso ${ }^{12}$, F. Pilo ${ }^{4}$, G. Piragino ${ }^{8}$, A. Regano ${ }^{1,5}$, \\ F. Riggi ${ }^{2}$, F. Romano ${ }^{1}$, G. Sartorelli ${ }^{7}$, E. Scapparone ${ }^{7}$, A. Scribano ${ }^{4}$, M. Selvi ${ }^{7}$, \\ S. Serci ${ }^{9}$, E. Siddi ${ }^{9}$, G. Spandre ${ }^{4}$, S. Squarcia ${ }^{12}$, M. Taiuti ${ }^{12}$, F. Toselli ${ }^{8}$, L. Votano ${ }^{3}$, \\ M.C.S. Williams ${ }^{5,7,13}$, A. Zichichi ${ }^{5,7,13}$, R. Zouyevski ${ }^{13}$ \\ ${ }^{1}$ INFN and Dipartimento di Fisica, Universitá di Bari, Bari, Italy \\ ${ }^{2}$ INFN and Dipartimento di Fisica e Astronomia, Universitá di Catania, Catania, Italy \\ ${ }^{3}$ INFN Laboratori Nazionali del Gran Sasso, Assergi (AQ), Italy \\ ${ }^{4}$ INFN and Dipartimento di Fisica, Universitá di Pisa, Pisa, Italy \\ ${ }^{5}$ Museo Storico della Fisica e Centro Studi e Ricerche E. Fermi, Roma, Italy \\ ${ }^{6}$ INFN Laboratori Nazionali di Frascati, Frascati (RM),Italy \\ ${ }^{7}$ INFN and Dipartimento di Fisica, Unversitá di Bologna, Bologna, Italy \\ ${ }^{8}$ INFN and Dipartimento di Fisica, Universitá di Torino, Torino, Italy \\ ${ }^{9}$ INFN and Dipartimento di Fisica, Universitá di Cagliari, Cagliari, Italy \\ ${ }^{10}$ INFN and Dipartimento di Fisica, Universitá di Salerno, Salerno, Italy \\ ${ }^{11}$ World Laboratory, Erice, Italy \\ ${ }^{12}$ INFN and Dipartimento di Fisica, Universitá di Genova, Genova, Italy \\ ${ }^{13}$ CERN, Geneva, Switzerland \\ ${ }^{14}$ INFN and Dipartimento di Fisica, Universitá di Lecce, Lecce, Italy
}

\begin{abstract}
The Extreme Energy Events project has been conceived to join the scientific interest of a real cosmic rays physics experiment with the enormous didactic potentiality deriving from letting it be carried out by high school students and teachers. After the initial phase, the experiment is starting to take data continuously, and the first interesting physics results have been obtained, demonstrating the validity of the idea of running a real physics investigation in these peculiar conditions. In this paper an overview of its structure and status is presented; also few results obtained at the sites are included.
\end{abstract}

XI workshop on Resistive Plate Chambers and Related Detectors - RPC2012, February 5-10, 2012 - INFN Laboratori Nazionali di Frascati Italy

\footnotetext{
* Speaker.

†e-mail: marcello.abbrescia@ba.infn.it
} 


\section{Introduction}

The Extreme Energy Event experiment [1] was conceived in 2003 by its scientific leader, prof. Antonino Zichichi, and it is presently carried out by Centro Fermi [2], in collaboration with INFN, CERN and MIUR (the Italian Ministry of Education, University and Research).

Its scientific goal is studying the most interesting, and still partially unexplored, region of the cosmic rays spectrum, i.e. the one whose primaries are characterized by an energy $>10^{18} \mathrm{eV}$. Here, issues related to the exact composition and origin, probably extragalactic, of this radiation, together with the precise shape of the spectrum (affected by presence of the Greisen, Zatspein and Kuz'min cutoff) still hold unsolved.

A standard technique to study this radiation consists in detecting the particles arriving at ground level - essentially muons - of the Extensive Air Showers (EAS) originated by the primaries when they enter the atmosphere. Since we are interested here to highly energetic primaries, the corresponding showers may impinge on the ground with millions of particles scattered on a several $\mathrm{km}$ radius area.

Therefore a great number of stations, distributed on a large surface, are needed. These stations, possibly, have to conjugate excellent performance in terms of efficiency, time and spatial resolution, with easiness of operation and low cost. The EEE collaboration choose to use a wider and cheaper version of the Multigap Resistive Plate Chambers (MRPC) already in use for the Time Of Flight system of the ALICE experiment at LHC, whose characteristics and performance in dedicated beam tests, described here [3], adequately fulfilled these requirements.

Each EEE station is composed by a telescope made of three MRPCs, and the detection of EAS is made by looking for muon events seen in coincidence among different stations. At regime, a network of about 100 stations all over Italy is foreseen.

The innovative aspect of the experiment is that the stations are located in Italian high school plus two at CERN - and are operated by teachers and students of these school, and that, moreover, the detector themselves have been built at CERN by the same teachers and students. The chambers were then sent to their institute of destination and the final installation and commissioning was performed by mixed teams under the supervision of experts and researchers from scientific institutions.

All this closely joins in the very same activity the important didactic aspect deriving from taking direct part to a real scientific experiment, with the interest of performing a research in an advanced field of investigation.

\section{Construction and characteristic of the stations}

The MRPCs specifically developed for the EEE project (see Fig. 1) are characterized by six gas gaps each, around $300 \mu \mathrm{m}$ thick, obtained by separating glass plates, $80 \times 160 \mathrm{~cm}^{2}$ in dimensions, by means of commercial nylon fishing lines used as spacers. The outer glass plates are coated with resistive paint, and act as high voltage electrode, while the inner ones are left electrically floating. Gas mixture is made out of $\mathrm{C}_{2} \mathrm{H}_{2} \mathrm{~F}_{4} / \mathrm{SF}_{6}$ mixed in $98 / 2$ proportion, and it is made flowing at a typical rate of $2-31 / \mathrm{h}[4]$. 


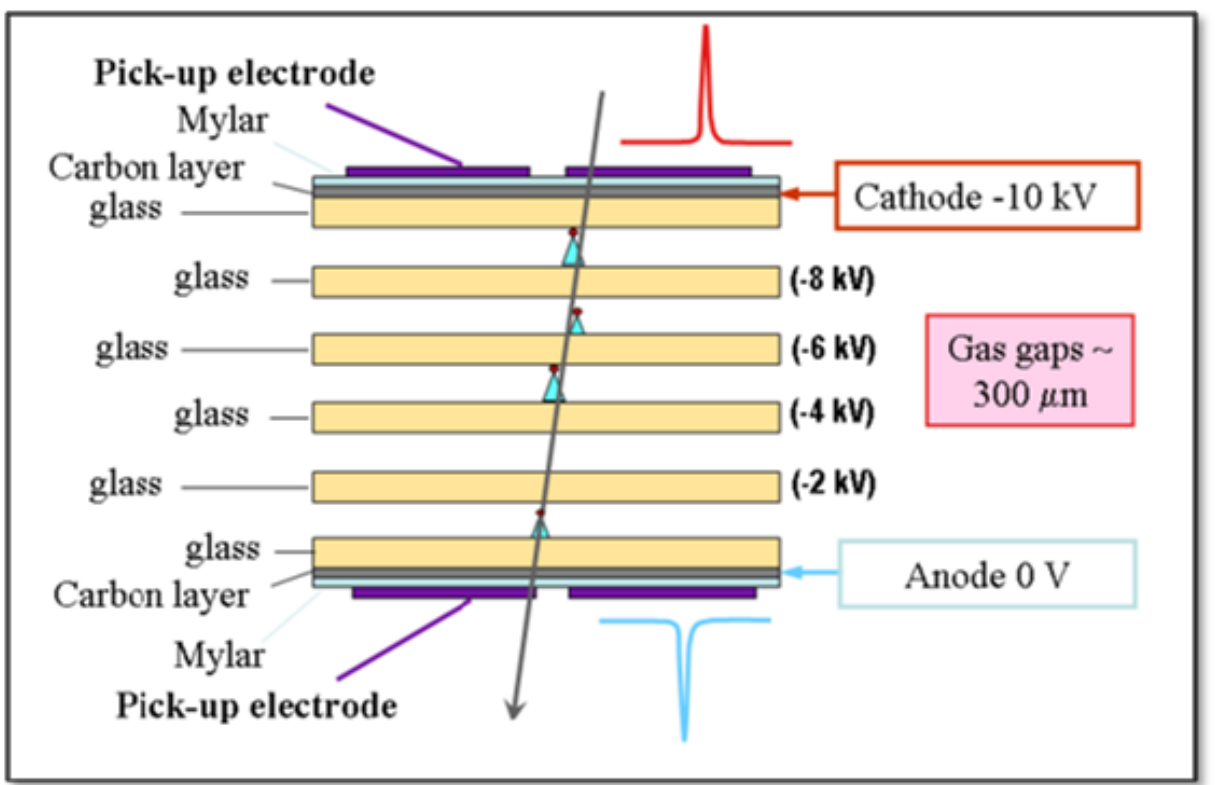

Figure 1: Layout of an MRPC used for the EEE experiment

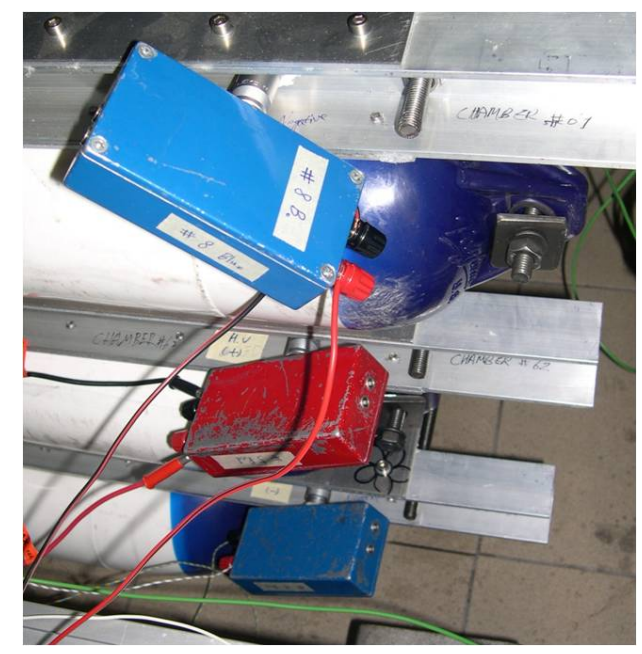

Figure 2: Picture of the HV box hosting the DC-DC converters, directly connected to the chamber electrodes

Standard operating voltage ranges around $18 \div 20 \mathrm{kV}$, so that the chambers operate in avalanche saturated mode, and the relative signals are induced on 24 copper strips (per chamber) glued on two vetronite plates placed on top and bottom of the glass outer electrodes. Signals are sent to NINOASIC chips based Front End electronics, amplified, discriminated and subsequently acquired by means of multi hit TDCs. Since readout strips lie longitudinally on the chambers, one coordinate $(x)$ of the muon impact point on the chamber is directly obtained from the fired strip, while the other $(y)$ by the difference of the signal arrival times at the two strip extremities.

The data acquisition system is based on VME standards, and the DAQ program is written in 


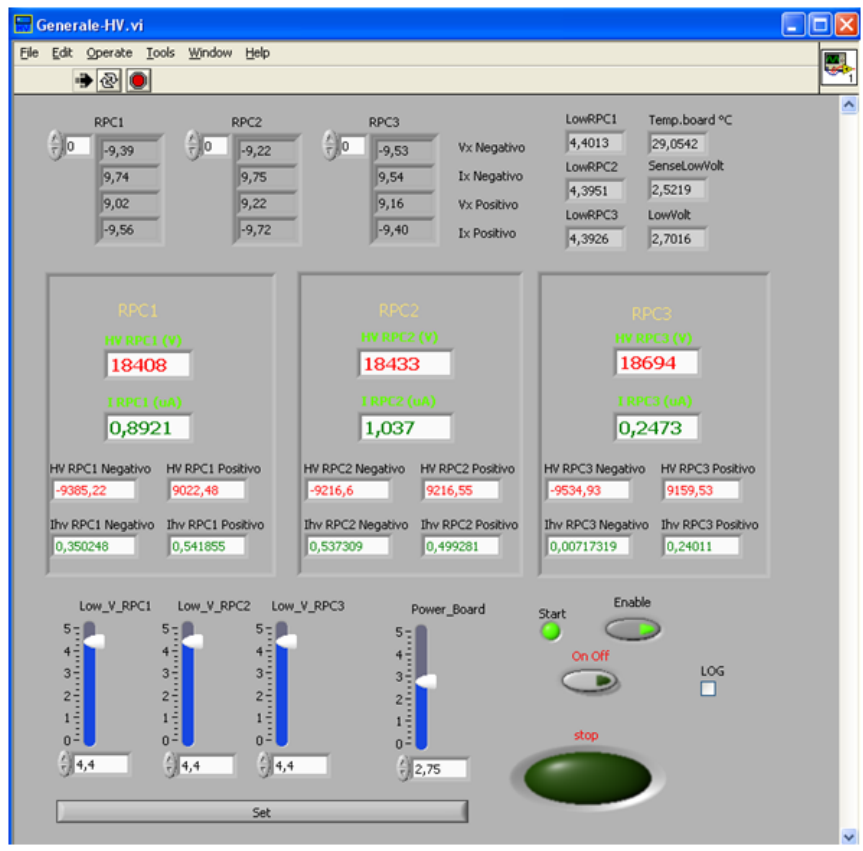

Figure 3: LV/HV Control panel of the EEE system

LabView and runs on a PC connected to the VME crate by means of a CAEN USB-VME bridge module. Since main goal of the EEE network is to detect coincidences in time among stations located at different positions, each event acquired must be provided by the relative time stamp; this is given by a Hytec Global Positioning System (GPS) VME module integrated in the system and readout by the DAQ program.

Since EEE stations operate in high schools, particular attention has been put on safety issues. For instance, the gas mixture does not contain any flammable component, like, for instance, isobutane, which is routinely used with this kind of chambers. High voltage is provided by small DC/DC converters of the EMCO-Q series, providing an output voltage up to $\pm 10 \mathrm{kV}$ when powered with 0-5 V, packed in small boxes and connected directly to the electrodes of the detector (see Figure 2).

Another peculiar aspect of the EEE network lies in the fact that the stations are under direct control of the students and teachers of the schools involved in the project. Nevertheless, from time to time some intervention or monitoring by the research personnel usually located in the nearby universities or local INFN sections is needed. This is the reason why most of the subsystems of the EEE telescope are directly monitorable or controllable from remote. For instance, high and low voltage power suppliers are interfaced with the DAQ PC and controlled by means of a dedicated software, and, since the PCs are permanently online, the LV/HV system can be continuously monitored from remote. In Fig. 3 the panel of the software control system for HV/LV is shown.

Moreover, it is important to keep under control the quality of the events taken, to be sure that the acquisition is running smoothly and the detector performance remains stable in time. A complete suite of online and offline monitoring programs has been set up for this important task, 


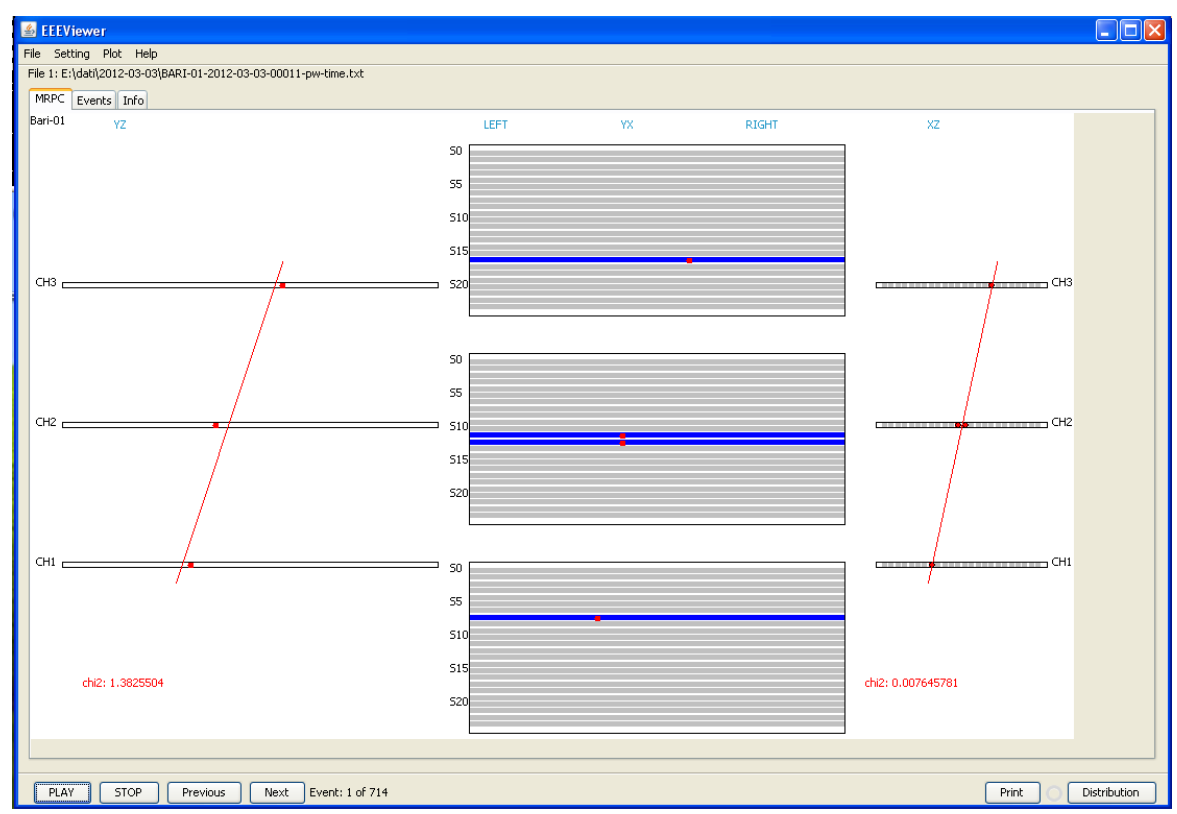

Figure 4: First event recorded at Liceo "A. Scacchi" in Bari

and is standardly used by teachers and students during their periodic checks of the stations.

Also an event display, based on java technology and therefore easily portable on different operating systems, has been developed. As an example of its potentialities, the first event acquired by the Liceo "A. Scacchi" in Bari, one of the schools recently joining the network, is shown in Fig. 4. The three orthogonal projections of the MRPCs, with indicated the strips fired and the computed positions along them, together with the reconstructed muon track, is displayed.

\section{Results}

One of the challenges of the EEE project was to obtain results of scientific validity when operating the EEE stations not in scientific laboratories, but in high schools. At the moment, the EEE collaboration has produced about 12 papers and around 20 contributions to conferences, mostly related to detector issues or the experiment structure and commissioning.

The most important parameter of the stations to keep under control is chamber efficiency. This cannot be monitored continuously, since the trigger requirement of the EEE stations during normal operation needs at least one signal coming from each of the six front-end boards per event, and therefore must be measured in dedicated periods. For this purpose, each chamber is in turn excluded by the trigger, and the line joining the two points of the remaining chambers is extrapolated on to the chamber under examination, searching for a hit there nearby. Efficiency vs. HV, measured in this way, for the three chambers located at the EEE station of Altamura is reported in Fig. 5, showing that it is over around $90 \%$ in all cases; since for these measurements just the coincidence between two chambers is required, an efficiency slightly lower than the one measured in dedicated beam tests is expected; nevertheless it remained stable in about three years of operation.

Muon trigger rate is also constantly kept under control, and it is shown, for a limited period during last October, in Fig. 6, for the same Altamura station already cited. Muon rate is affected 


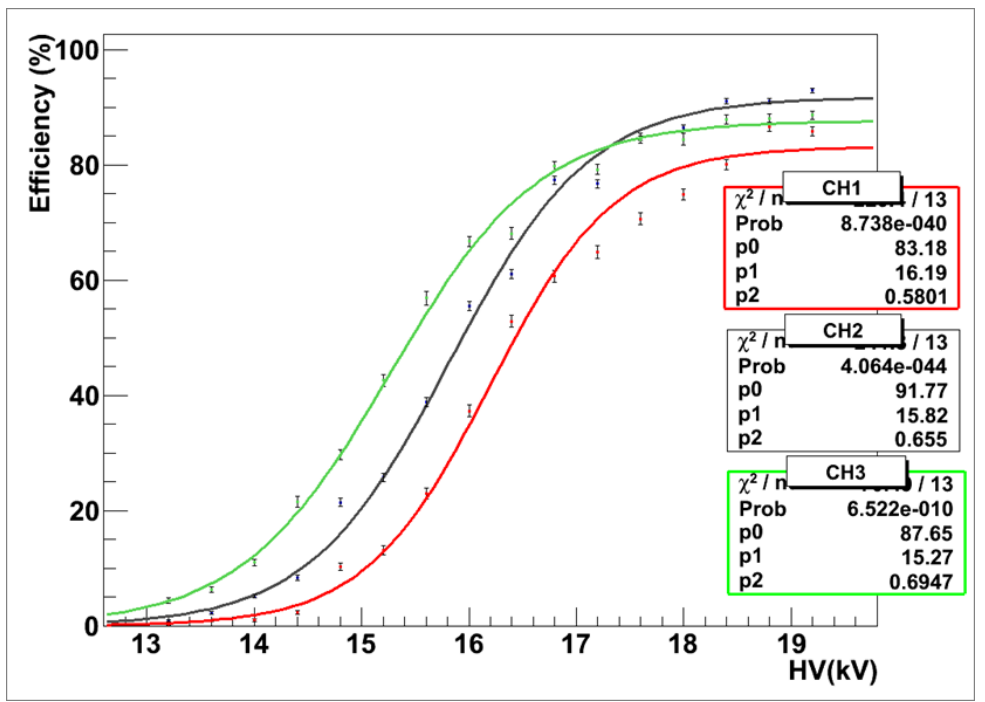

Figure 5: Efficiency vs. HV of the three chambers located at the EEE station of Altamura

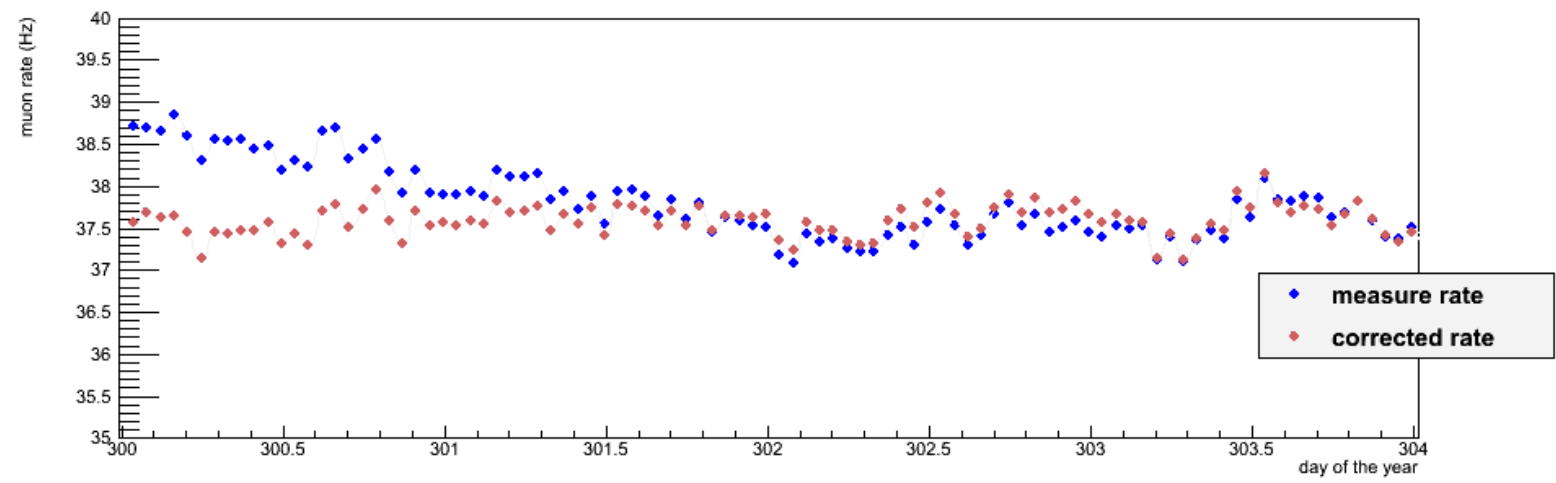

Figure 6: Muon trigger rate of the EEE telescope located at Altamura, during few days of last October

by environmental conditions, because of the barometric effect related to muon absorption in the atmosphere, and because changes in gas pressure have a direct impact on the avalanche development inside the MRPC. Therefore, a correction factor - in this case of around $0.40 \% / \mathrm{mbar}$ - was applied to the measured rate; the corrected rate is shown in Fig. 6 too, and it shows a much more stable behavior with respect to the uncorrected measured rate, as expected.

In addition to studies related to detector behavior, the possibility to obtain interesting physics results was definitely put in evidence when the first coincidences between different stations of the EEE network were recorded, demonstrating the ability of detecting EAS with the EEE stations. Due to the expected frequency for observing coincidences, the first data came from the closest stations of the experiment, distant only about $200 \mathrm{~m}$, and located at L'Aquila, whose results were already published [5].

Moreover, during last February 2011, a class X2 solar flare, followed by an important Coronal Mass Emission (CME) gave origin to a Forbush decrease recorded by some of the stations of the Neutron Monitor Network (NMN). Two stations of the EEE network saw a similar decrease in the 
muon rate, with an intensity profile clearly comparable to the ones observed by the stations of the NMN [6].

This kind of observations are intrinsically interesting, since X-class flares can trigger even blackouts on Earth, or, in general, affect the space weather conditions close to our planet. To our knowledge, this has been the first observation of a Forbush decrease performed by means of detectors installed in high schools, outside of scientific laboratories, and, for this particular event, the first to be published. Nevertheless, the results obtained show an overall quality comparable to the ones obtained in professional observatories.

\section{Conclusions}

The EEE experiment is an innovative approach both to scientific research and scientific communication. Its stations, located in high schools all over Italy and at CERN, acquire data almost continuously, and their operation relies on the incredible enthusiasm shown by students and teachers.

In addition to its invaluable didactic functions, EEE produces interesting physics results; its detectors show a performance, in terms of efficiency and time resolution, comparable to the one characteristics of similar detectors installed at the LHC experiments.

The first coincidences among nearby stations have already been recorded, demonstrating the possibility of effectively revealing Extensive Atmospheric Shower using the EEE array. Finally, the detection of the February 2011 Forbush decrease has opened up new and interesting possibilities to use the EEE network also for studying the solar activity and its effects on the cosmic ray muon flux on Earth.

\section{References}

[1] R. Antolini et al, The EEE Project, 29th ICRC Proceedings, Pune 8, (2005) 279

[2] Centro Fermi web site: www.centrofermi.it

[3] M. Abbrescia et al., Performance of a six gap MRPC built for a large area coverage, Nucl. Inst. and Meth. in Phys. Res., A 539 (2008) 263-286

[4] M. Abbrescia et al, Extreme Energy Events Project: Construction of the detectors and installation in Italian High Schools, Nucl. Instr. and Meth A 588 (2008), 211-214

[5] M. Abbrescia et al., First detection of extensive air showers with the EEE experiment, Il Nuovo Cimento, Vol. 125 B, 243 (2010)

[6] M. Abbrescia et al., Observation of the February 2011 Forbush decrease by EEE telescopes, Eur. Phys. J. Plus (2011)126: 61 